\title{
Role of miR-23a/Zeb1 negative feedback loop in regulating epithelial-mesenchymal transition and tumorigenicity of intraocular tumors
}

\author{
YANYAN WANG, YUNNA LUO, WENYING GUAN and HAIXIA ZHAO \\ Department of Ophthalmology, Affiliated Hospital of Inner Mongolia Medical University, \\ Hohhot, Inner Mongolia 010059, P.R. China
}

Received September 18, 2017; Accepted May 23, 2018

DOI: $10.3892 / 01.2018 .8940$

\begin{abstract}
Role of the two-way negative feedback regulation channel formed by miR-23a and Zeb1 in epithelial-mesenchymal transition (EMT), tumorigenic ability, and migration and metastasis capacity of the intraocular malignant tumor cells was investigated. Molecular biological methods such as real time-quantitative PCR (RT-qPCR), immunoblotting method, and immunofluorescence were used to detect the expression levels of mRNA and protein in the Zeb1 factor in OCM-1, WERI-RB1, and Y79 cells before and after miR-23a transfection. Transwell cells were used to detect the in vitro membrane permeation and migration ability in OCM-1, WERI-RB1, and Y79 cells (non-transfection group, blank control transfection group, mimic transfection group, inhibitor transfection group). The results revealed that the relative expression of miR-23a in the cells in the miR-23a mimic transfection group increased significantly compared with that in the control group $(p<0.05)$. There were significant differences in the relative expression of mRNA between the mimic transfection and control group $(\mathrm{p}<0.05)$. RT-qPCR detection showed that the relative expression of mRNA of the epithelial-labeled factor E-cadherin increased significantly in the miR-23a mimics group ( $<<0.05)$. Expression of the protein E-cadherin increased while the expression of the mesenchyme-labeled proteins of vimentin and $\mathrm{N}$-cadherin decreased in the mimics group. Zeb1 has a negative feedback effect on miR-23a. They can form a negative feedback loop. The results showed that miR-23a and Zeb1 form a bidirectional inhibitory negative feedback loop, which plays an important role in regulating EMT. In conclusion, the significant changes in the
\end{abstract}

Correspondence to: Dr Haixia Zhao, Department of Ophthalmology, Affiliated Hospital of Inner Mongolia Medical University, 1 Tongdao North Street, Huimin District, Hohhot, Inner Mongolia 010059, P.R. China

E-mail: hae2pw@163.com

Key words: miR-23a, Zeb1, epithelial-mesenchymal transition, choroidal melanoma, retinoblastoma mesenchymal phenotype of the stable strains with Zeb1 overexpressed in the OCM-1 cells cannot be completely explained with the changes in cytoskeleton caused by EMT.

\section{Introduction}

The core of epithelial-mesenchymal transition (EMT), is to lose the epithelium-derived labelled protein E-cadherin and acquire the labeled protein of the mesenchymal cells (such as vimentin, $\mathrm{N}$-cadherin) (1). E-cadherin is one of the important components for adherent junction of the epithelial cells. It is bound to the intracellular proteins to form morphology, polarity, and functions of the epithelial cells thus allowing for tight junctions between epithelial cells and maintaining the integrity of the structure of the epithelial tissue (2). In addition to the decreased expression of the labeled E-cadherin, the EMT can also lead to changes in other epithelium-derived characteristic phenotypes, including tight junctions, gap junctions between epithelial cells, and desmosome (3). Accordingly, interstitial cytoskeletal reorganization occurs in the cells with EMT.

Currently, a great deal of research shows that several transcription factor families can directly act on the E-cadherin promoter and directly inhibit its transcription. These transcription factors include: zinc finger protein family Zeb (Zeb1 and Zeb2) (4), Snail family (Snail1, Snail2, and Snail3) (5), Twist family (Twist1 and Twist2) (6), fork-head (7) transcription factor family FOXC2 (8) and other transcription factors under investigation. The Zeb family is a generally-accepted EMT activated transcription factor including Zeb1 and Zeb2. Zeb1 can directly inhibit the expression of E-cadherin (9) and initiate the EMT process by means of combination between zinc finger and E-box in the E-cadherin genetic promoter region. Moreover, in addition to E-box, research showed that the action of Zeb1 on E-cadherin may also have other mechanisms. The compound formed by Zeb1 and the histone deacetylase may participate in the transcription splicing of E-cadherin thus inhibiting its genetic expression (10). Downregulation of the expression of Zeb1 in mesenchymal tumor cells may lead to mesenchymal-epithelial transition in tumor cells and epithelial cell phenotype in cells (11). Research shows 
that Zeb1 can significantly increase the invasiveness and metastasis rate of tumors by inducing the EMT process of the tumor cells (12).

MicroRNA is a class of short non-coding RNA comprising only 20-22 nucleotides. They silence the target gene or inhibit their translation by specifically binding and cutting the messenger RNA (13). A great deal of research shows that the miR-23a family plays a role in inhibiting the EMT of tumor cells thus leading to opposite mesenchymal-epithelialization transition and promoting the expression of E-cadherin (14). Transfecting the mesenchymal pancreatic carcinoma and breast carcinoma with the miR-23a precursor allows for transition from mesenchyme to epithelium, increased expression of E-cadherin, decreased expression of vimentin, and occurrence of epithelial phenotype in cells (15). In addition, research on the ovarian carcinoma cells indicates that the overexpression of miR-23a can significantly inhibit the migration ability of the cancer cells and strengthen their sensitivity to the chemotherapeutic drugs. On the contrary, overexpression of miR-23a can significantly decrease the tumorigenic ability of pancreatic carcinoma cells (16).

miR-23a and Zeb1 have completely contrary functions in regulation of EMT. Moreover, they also have diametrically opposite functions in regulation of such characteristics as stemness maintenance in the tumor stem cells, and drug resistance, related to EMT. The former decreases the tumorigenic ability of the tumor cells and increases their sensitivity to chemotherapy. The latter increases the cellular proliferation ability and improves drug resistance. Furthermore, direct mutual inhibition effect also occurs. Therefore, Zeb1 and miR-23a form a bidirectional inhibitory negative feedback loop. The roles of Zeb1-miR-23a in regulating EMT of tumor cells, tumor deterioration, metastasis, drug resistance, and recurrence are arousing wide attention to some human malignant tumors (such as pancreatic, bladder, breast, and thyroid carcinoma) (17). However, there is still lack of research on ocular malignancies. Choroidal melanoma and retinoblastoma are common intraocular tumors with a high morbidity, a malignant grade, and a high susceptibility to metastasis. Particularly, hematogenous metastasis has a high case fatality rate as it occurs in the remote organ in an early stage of choroidal melanoma. A probe into the mechanism of metastasis of these malignant tumors makes it possible to identify new strategies to better control and treat these tumors and decrease their case fatality rates.

To further test the feedback regulation effect of Zeb1 on miR-23a, we establish the Zeb1 overexpression lentiviral vector to achieve stable transfection of the human choroidal melanoma cell strain OCM-1. Then, we utilized the expression of the puromycin resistance gene carried by the Zeb1 overexpression lentiviral vector and used the puromycin to screen for the stable transfected strains from the OCM-1 cells transfected with lentivirus to obtain the stable strain cells with overexpression of Zeb1 thus laying a foundation for subsequent establishment of the animal models and the in vivo experiments of tumorigenicity and metastasis ability of tumor cells. Based on the real time-quantitative PCR (RT-qPCR) detection and western blot analysis, we quantitatively detected the cells in the control and Zeb1 overexpression group for Zeb1 and miR-23a and the role of overexpression of Zeb1 in the cell strain in regulating miR-23a. We also further detected the related factors and stemness factors of the EMT to evaluate the role of Zeb1 in regulating EMT and stemness tumorigenicity.

\section{Materials and methods}

Transfection of liposome-mediated small RNA mimics and inhibitors. At 1 day before transfection, $4.5 \times 10^{4}$ cells were inoculated into a 6 -well microplate and cultured in a $5 \%$ $\mathrm{CO}_{2}$ incubator at $37^{\circ} \mathrm{C}$ after addition of $2 \mathrm{ml}$ of basal culture medium containing fetal bovine serum (Invitrogen: Thermo Fisher Scientific, Inc., Carlsbad, CA, USA). The mixture of miR-23a mimics or inhibitor and Lipofectamine ${ }^{\mathrm{TM}} 2000$ (all from Invitrogen: Thermo Fisher Scientific, Inc.) was added to each well containing cells and culture medium. The culture plate was shaken gently back and forth to mix well the solution and the cell culture medium. The cells were placed in a $\mathrm{CO}_{2}$ incubator at $37^{\circ} \mathrm{C}$. The transfection efficiency was determined with fluorescence at $6 \mathrm{~h}$ (Takara Bio, Inc., Tokyo, Japan). It was replaced by the standard serum-containing culture medium at $12 \mathrm{~h}$. The cells were collected for further detection after they were incubated for $48 \mathrm{~h}$ in the $5 \% \mathrm{CO}_{2}$ incubator at $37^{\circ} \mathrm{C}$. The study was approved by the Ethics Committee of the Affiliated Hospital of Inner Mongolia Medical University (Hohhot, Inner Mongolia, China).

$R T-q P C R$. A total of $10^{6}$ intraocular tumor cells were directly added to $1 \mathrm{ml}$ TRIzol, mixed well on a vortex mixer, and allowed to stand for $5 \mathrm{~min}$ at room temperature. After rotation, the supernatant was transferred to a new tube and the same volume of isopropanol was added (Beijing Chemical Reagents Co. Ltd., Beijing, China), the solution was mixed well for $1 \mathrm{~min}$ by turning upside down, and allowed to stand for $5 \mathrm{~min}$ at room temperature and centrifuged for $15 \mathrm{~min}$ at $8,000 \mathrm{x} \mathrm{g}$ at $4^{\circ} \mathrm{C}$. After addition of isopropanol of the same volume (Beijing Chemical Reagents Co., Ltd.), the cells were mixed well by gently turning upside down, and allowed to stand for $10 \mathrm{~min}$ at room temperature and centrifuged for $10 \mathrm{~min}$ at $8,000 \mathrm{x} \mathrm{g}$ at $4^{\circ} \mathrm{C}$. The supernatant was discarded and $1 \mathrm{ml}$ of $75 \%$ ethanol was added. An appropriate volume of DEPC water was added (EMD Millipore, Billerica, MA, USA) to sufficiently dissolve the sediment. The reaction system (Takara Bio, Inc.) was $25 \mu \mathrm{l}$ : fluorescence RT-qPCR reaction solution $20 \mu \mathrm{l}$, DNA polymerase $1 \mu \mathrm{l}$, reverse transcriptase $0.35 \mu \mathrm{l}$, template RNA $5 \mu 1$, mixed well and centrifuged for $10 \mathrm{sec}$ at 3,000 x g. RT-qPCR amplification procedures: reversely transcribed for $30 \mathrm{~min}$ at $50^{\circ} \mathrm{C}$; pre-denatured for $3 \mathrm{~min}$ at $95^{\circ} \mathrm{C}$; denatured at $95^{\circ} \mathrm{C} ; 15 \mathrm{sec}$, annealed for $30 \mathrm{sec}$ at $50^{\circ} \mathrm{C}$, extended for $30 \mathrm{~min}$ at $72^{\circ} \mathrm{C}, 5$ cycles in total; denatured for $10 \mathrm{sec}$ at $95^{\circ} \mathrm{C}$, annealed for $40 \mathrm{sec}$ at $55^{\circ} \mathrm{C}, 40$ cycles in total. The primer sequence was obtained from previous studies $(18,19)$.

Western blot analysis of protein expression. OCM-1, WERI-RB1, and Y79 cells in the logarithmic growth phase were diluted and inoculated into a 6-well microplate for culture. The cells in the control and treatment group were collected, respectively, and subjected to RIPA lysis (Promega Corp., Madison, WI, USA), protein quantification, and western blot analysis. The cells were first subjected to SDS-polyacrylamide gel electrophoresis. The protein specimen was transferred 
to the nitrocellulose (NC) membrane (Polysciences, Inc., Warrington, PA, USA), and blocked. The Zeb1 antibody $(1: 1,000)$, E-cadherin (both from Cell Signaling Technology, Inc., Danvers, MA, USA), N-cadherin, vimentin (both from Abcam, Cambridge, UK) antibodies were incubated (dilution rates, 1:1,000). The internal reference was $\beta$-actin $(1: 2,000$; Abcam). The cells were incubated for $2 \mathrm{~h}$ at room temperature and incubated with the secondary antibody for $1 \mathrm{~h}$. The chemiluminescence method was used.

Immumofluorescence method. The OCM-1, WERI-RB1, and Y79 cells were rinsed 3 times for 5 min each with PBS after growing on the glass slide. The cells were blocked for $20 \mathrm{~min}$ at $37^{\circ} \mathrm{C}$ after the goat serum working solution was added dropwise. The cells were allowed to stand overnight at $4^{\circ} \mathrm{C}$ and rinsed 3 times for 5 min each with PBS next day after the goat anti-Zeb1 (1:500): rabbit anti-E-cadherin (1:500) (both from Cell Signaling Technology, Inc.), mouse anti-vimentin (1:500; Abcam) were added dropwise. The cells were incubated for $2 \mathrm{~h}$ at $37^{\circ} \mathrm{C}$ after the specific secondary antibody Alexa Fluor 594 $(1: 1,000)$ and Alexa Fluor 488 (1:1,000) (both from Invitrogen: Thermo Fisher Scientific, Inc.) were added dropwise. The cells were rinsed 3 times for $5 \mathrm{~min}$ each. The nuclei were stained with DAPI. The cells were air dried, mounted with anti-fluorescence quenching mounting solution, and preserved in the dark at $4^{\circ} \mathrm{C}$ for microscopic examination.

Cell migration experiment (Transwell). Trypsin was added to the culture flask to digest the cells. After the digestion was terminated, the cell suspension was pipetted, added to a centrifugal tube, and centrifuged for $5 \mathrm{~min}$ at $3,000 \mathrm{x} \mathrm{g}$. The cells were washed once or twice with PBS and resuspended with the serum-free medium containing BSA until the cell density reached $1 \times 10^{4} / \mathrm{ml}$ after the culture solution was discarded. The cells and the culture medium were inoculated into a Transwell 6-well microplate (Corning, Inc., Corning, NY, USA). A total of $1 \mathrm{ml}$ of the culture medium containing FBS was added to the lower chamber. Then, $2 \mathrm{ml}$ of the serum-free tumor cell suspension prepared in advance was added to the upper chamber. The cells were routinely cultured for $24 \mathrm{~h}$. The culture medium in the upper chamber was discarded. The cells on the upper chamber side of the semipermeable membrane at the bottom were removed with a cotton swab. The cells were fixed for 15 min with $95 \%$ ethanol, stained for 10 min with hematoxylin (Takara Biotechnology Co., Ltd., Dalian, China), and observed and photographed under an inverted microscope using four fields of view (x200). The cells passed through the semipermeable membrane and entered the lower chamber. The cell migration ability was detected.

Lentivirus transfection. One day before transfection, the cell suspension with a density of $5 \times 10^{4}$ was inoculated into 3 wells of the 6-well microplate and allowed to stand overnight in an incubator. The old culture medium was discarded the following day. The cells were washed once with the D-Hank's solution. Then, $2 \mathrm{ml}$ of the freshly-prepared $7 \%$ non-resistant culture medium Opti-MEM I (Invitrogen: Thermo Fisher Scientific, Inc.) was added. The first well served as the normal group and the second well served as the experimental group. A total of $30 \mu \mathrm{l}$ Zeb1 virus solution and $2 \mu \mathrm{l}$ transfection solution polybrene (Takara Bio, Inc.) were added in the experimental group. The solution was mixed well and placed in the incubator. The cellular growth was observed every other day and the solution was replaced every third day. The OCM-1 cells were collected and transferred to a 24-well microplate with a density of $5 \times 10^{4}$ cells after being transfected for $72 \mathrm{~h}$ with lentivirus. The common DMEM culture medium containing serum was added. The cells were incubated overnight. The screening culture medium was prepared. The fresh culture medium contained $1.25 \mu \mathrm{g} / \mathrm{ml}$ puromycin (Biosino Bio-Technology and Science Inc., Beijing, China). The prepared puromycin screening culture medium was used to replace the old culture medium. The old culture medium was replaced with the freshly-prepared screening culture medium at $\sim 12-24 \mathrm{~h}$ after cell death. Apoptotic cells were removed. To minimize the changes in cellular phenotype arising from puromycin, the puromycin culture medium was removed and replaced with the normal culture medium at $72 \mathrm{~h}$. The survived cells were considered as stably transfected cells.

Statistical analysis. The statistical software SPSS 19.0 (IBM Corp., Armonk, NY, USA) was used for statistical analysis. Excel was used for statistical charting. ANOVA was used for comparison of multiple groups and the post hoc test was LSD. The differences were considered statistically significant at $\mathrm{P}<0.05$.

\section{Results}

Role of miR-23a transfection in regulating the expression of the target gene Zeb1. RT-qPCR was used to detect the expression levels of miR-23a before and after transfection of cells. The results showed (Fig. 1A) that the relative quantitative (RQ) values of the non-transfection control group of the OCM-1, WERI-RB1, and Y79 cell strains were 2.83, 3.28 , and 2.3 , respectively. The relative expression levels of miR-23a in the three groups of cells were $6.89,7.74$, and 5.86 , respectively, after the cells were transfected with the hsa-miR-23a mimic, which increased significantly compared with those in the non-transfected control group $(\mathrm{p}<0.05)$. The relative expression levels of miR-23a in the three intraocular tumor cell strains were $0.19,0.18$, and 0.33 , respectively, after the cells were transfected with the hsa-miR-23a inhibitor, which decreased significantly compared with those in the control group $(\mathrm{p}<0.05)$. RT-qPCR results showed that the mRNA RQ values of Zeb1 in the non-transfected control group of the OCM-1, WERI-RB1, and Y79 cell strains were OCM-1: 3.30, WERI-RB1: 4.45, and Y79: 2.74. The mRNA RQ values of Zeb1 of the three cell strains were OCM-1: 1.07, WERI-RB1: 1.17, and Y79: 1.14 after they were transfected with the hsa-miR-23a mimic, which decreased significantly compared with those in the non-transfected control group $(\mathrm{p}<0.05)$ (Fig. 1B). The cells in the miR-23a mimic transfection group, the miR-23a inhibitor transfection group, and the non-transfection group of the human choroidal melanoma cell strains OCM-1, human retinoblastoma cell strains WERI-RB1, and Y79 were collected and subjected to protein immunoblotting for determination of the changes in expression of Zeb1 at the level of protein (Fig. 1C). The immunofluorescence assay of Zeb1 in the three intraocular 
A

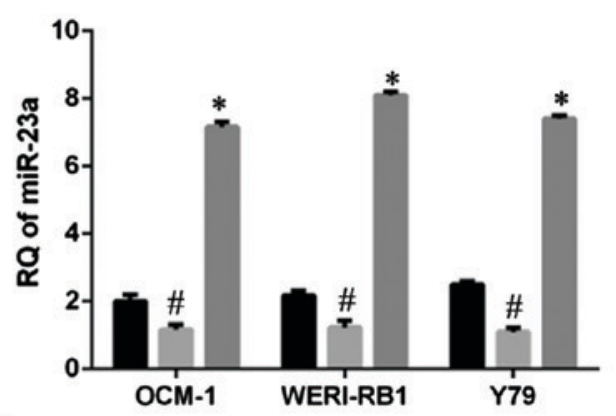

B

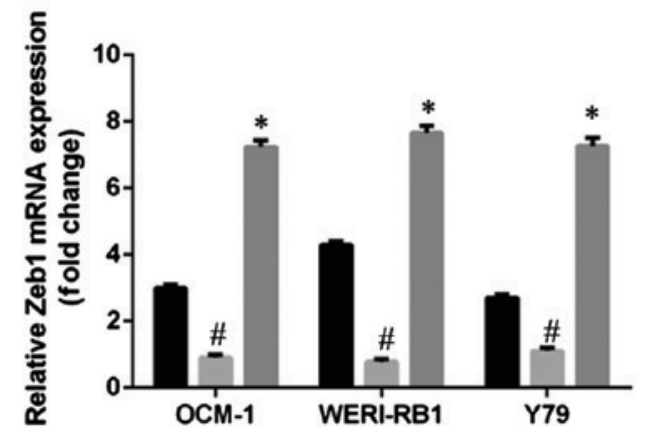

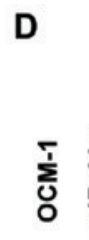
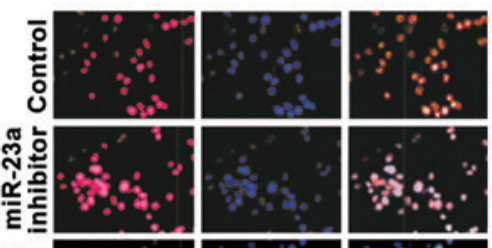

Inhibitor

Mimics
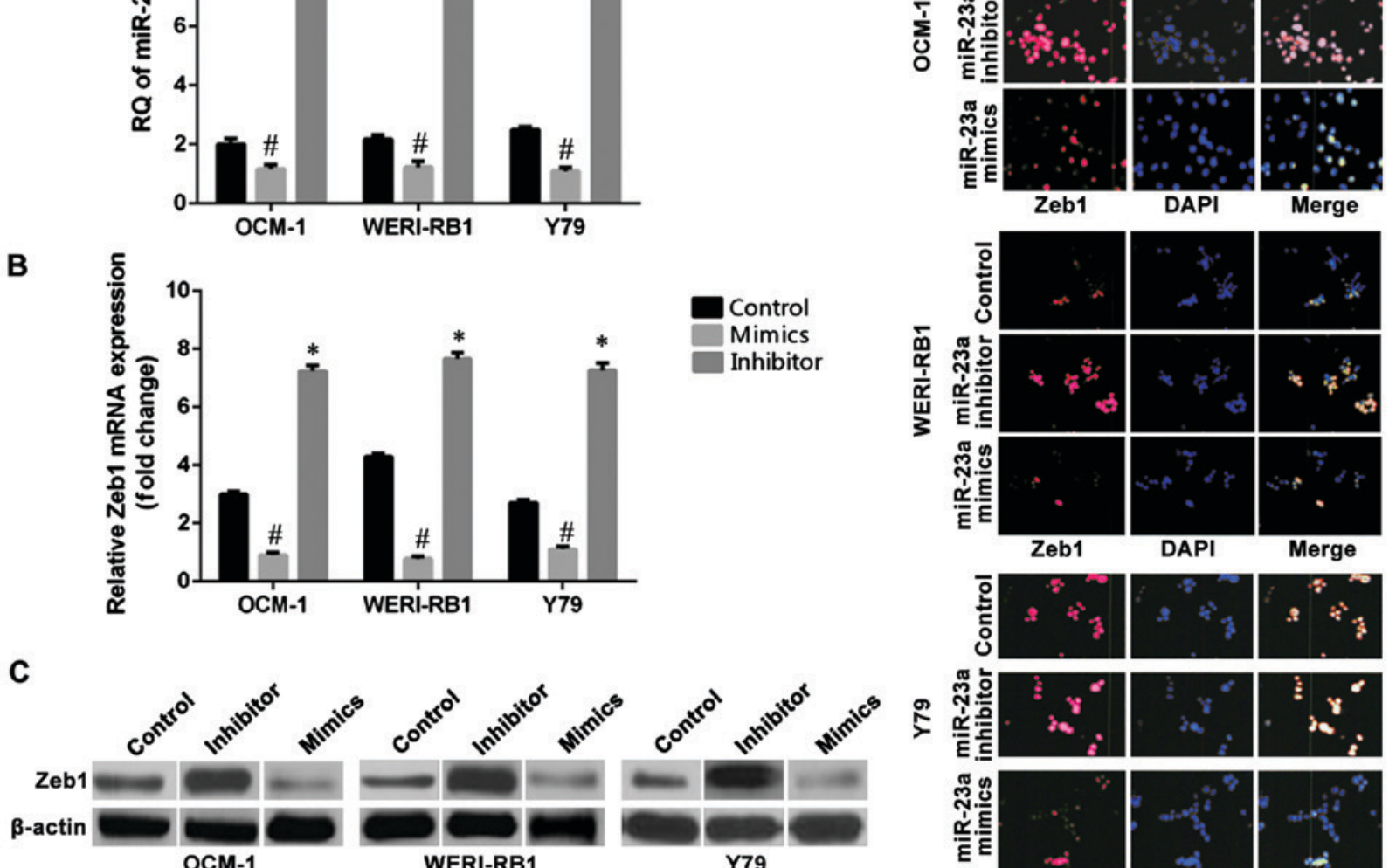

OCM-1
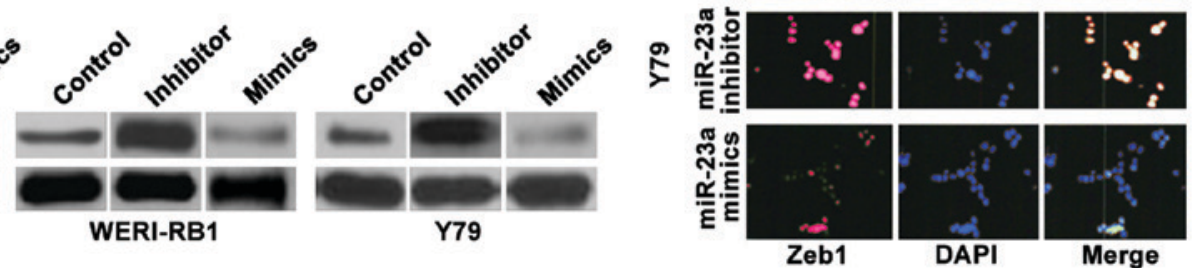

Figure 1. Role of miR-23a transfection in regulating the expression of the target gene Zeb1. (A) In terms of the three intraocular malignant tumor cell strains, the expression level of miR-23a in the mimic transfection group increased significantly compared with that in the control group $(\# \mathrm{p}<0.05)$ while the inhibitor transfection downregulated the expression of miR-23a ( $\left.{ }^{*} \mathrm{p}<0.05\right)$. (B) The miR-23a mimic transfection downregulated the mRNA expression levels of Zeb1 in the three tumor cells $\left({ }^{\#} \mathrm{p}<0.05\right)$ while the miR-23a inhibitor transfection enabled the mRNA expression level of Zeb1 to increase ( $\left.{ }^{*}<0.05\right)$. (C) The expression of the Zeb1 protein in the miR-23a inhibitor transfection group increased compared with that in the non-transfection group while the expression of the Zeb1 protein in the mimic transfection group decreased compared with that in the non-transfection group. (D) In terms of the immunofluorescent expression of the Zeb1 antibody after transfection of the miR-23a in the human choroidal melanoma cell strain OCM-1, human retinoblastoma cell strain WERI-RB1, and Y79, the transfection of the miR-23a mimic decreased the expression level of Zeb1 while the inhibitor transfection increased the expression level of Zeb1 within the tumor cells.

tumor cell strains were OCM-1: 6.99, WERI-RB1: 7.81, and Y79: 6.92 after the cell strains were transfected with the hsa-miR-23a inhibitor, which increased significantly compared with those in the control group $(\mathrm{p}<0.05)$ (Fig. 1D).

Role of miR-23a transfection in regulating the EMT in intraocular tumors. RT-qPCR was used to detect the expression levels of mRNA in the epithelial cell-labeled gene E-cadherin and the mesenchyme-labeled gene vimentin in the human choroidal melanoma cell strains OCM-1 (Fig. 2A-C), human retinoblastoma cell strains WERI-RB1 (Fig. 2D-F), and the Y79 cells (Fig. 2G-I) before and after miR-23a transfection. The results showed that the relative expression levels of mRNA of E-cadherin in the three strains of cells following the hsa-miR-23a mimic transfection increased significantly compared with those in the non-transfected control group $(p<0.05)$ while the expression levels of vimentin at the level of RNA decreased significantly $(\mathrm{p}<0.05)$. Also, opposite changes occurred in the miR-23a inhibitor groups of the three strains of cells. The expression of E-cadherin decreased significantly compared with that in the control group $(\mathrm{p}<0.05)$ while the expression of vimentin increased significantly.
Effect of miR-23a transfection on the migration capacity of the intraocular tumor cells. The cells in the non-transfected control group, negative miR transfection control group, miR-23a mimic transfection group, and the miR-23a inhibitor transfection group of the human choroidal melanoma cell strains OCM-1, and human retinoblastoma cell strains WERI-RB1 and Y79 were added to the Transwell 6-well microplate. The cells that permeated the membrane and entered the lower chamber were photographed and counted under high power lens. The results showed that the average counts of the membrane-permeating cells in the non-transfected control group were: OCM-1: 54.75, WERI-RB1: 49.75, and Y79: 66.75. The average counts of the cells in the negative transfection control group were: OCM-1: 57.5, WERI-RB1: 55.25: Y79: 68.25. The differences between the two groups were not statistically significant. The average counts of the membrane-permeating cells in the miR-23a mimic transfection group were: OCM-1: 25.75, WERI-RB1: 28.25, Y79: 32.5, which decreased when compared with those in the non-transfection group $(\mathrm{p}<0.05)$ and negative control group $(p<0.05)$. The average counts of the cells in the miR-23a inhibitor transfection group were: OCM-1: 153.5; WERI-RB1: 165.25; Y79: 145, which increased significantly 
A

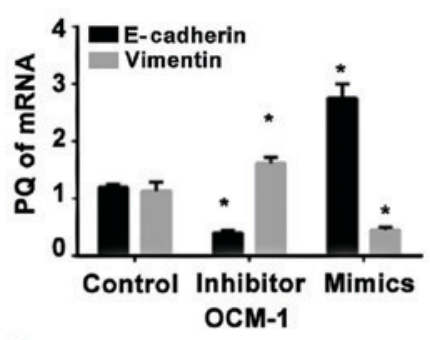

D

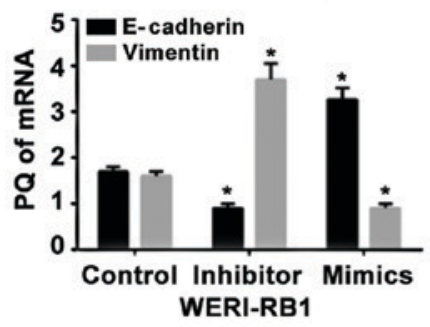

G

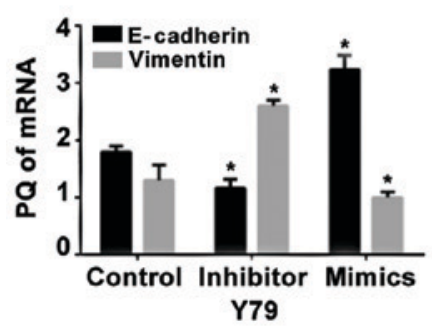

B

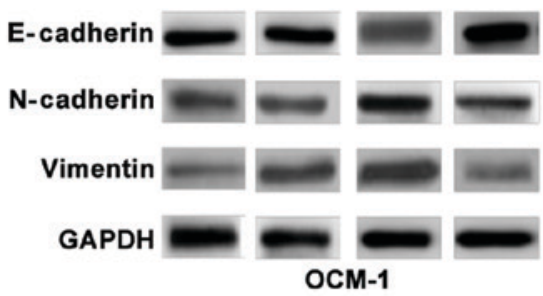

E

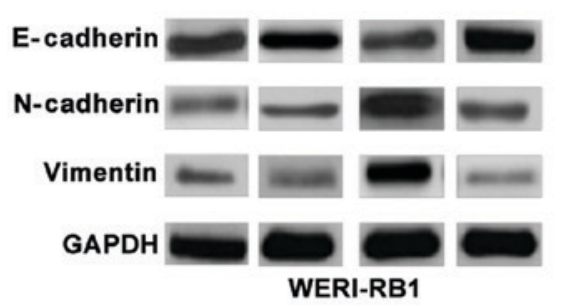

H

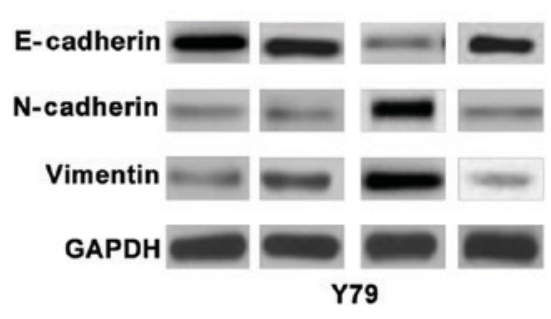

C
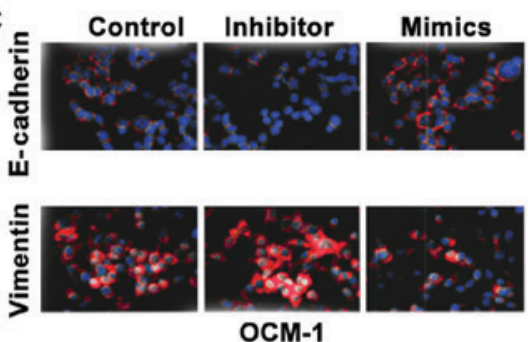

F
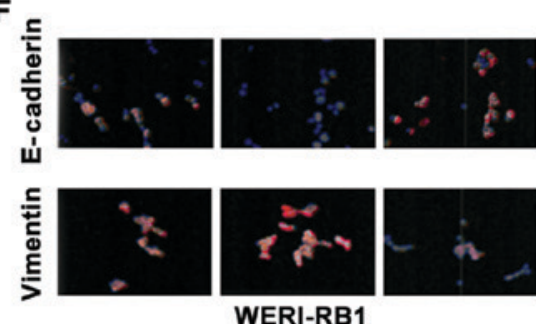

WERI-RB1

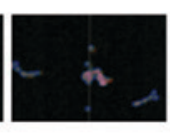

I

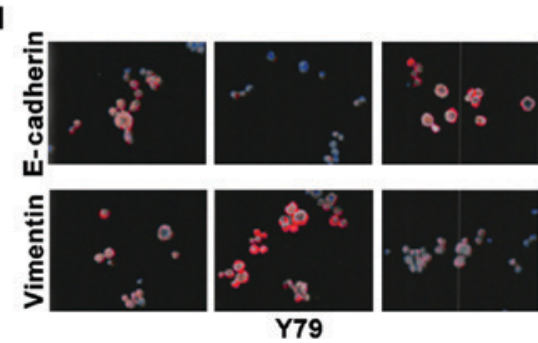

Figure 2. Role of miR-23a transfection in regulating the EMT in intraocular tumor cells. RT-qPCR detection of the role of miR-23a transfection in (A) human choroidal melanoma cell strains OCM-1, (D) human retinoblastoma cell strains WERI-RB1 and (G) Y79 in regulating the expression of E-cadherin at the level of mRNA. The inhibitor transfection of miR-23a significantly decreased the mRNA of E-cadherin relative to the control group ("p<0.05) while vimentin increased significantly ( $\left.{ }^{*} \mathrm{p}<0.05\right)$. The mimic transfection significantly increased E-cadherin $\left({ }^{*} \mathrm{p}<0.05\right)$ and significantly decreased vimentin $\left({ }^{*} \mathrm{p}<0.05\right)$. Western blot analysis was used to detect the effect of miR-23a transfection of (B) human choroidal melanoma cell strains OCM-1, (E) human retinoblastoma cell strains WERI-RB1 and (H) Y79 on the expression of the proteins related to EMT. The miR-23a mimic transfection increased the expression of the epithelial cell marker, E-cadherin protein whereas the expression levels of the mesenchymal cell-labeled proteins of N-cadherin and vimentin decreased significantly. The miR-23a inhibitor transfection group exhibited an opposite acting trend. The proteins related to EMT were subjected to an immunofluorescence analysis after (C) the human choroidal melanoma cell strains OCM-1, (F) the human retinoblastoma cell strains WERI-RB1 and (I) Y79 were transfected with miR-23a. The expression of the epithelial marker E-cadherin in the inhibitor transfection group decreased while the expression of the mesenchyme-labeled vimentin increased. Vimentin decreased and E-cadherin increased in the mimic transfection group. EMT, epithelial-mesenchymal transition.

when compared with those in the non-transfection group $(\mathrm{p}<0.05)$ and the negative control group $(\mathrm{p}<0.05)$. Inhibition of miR-23a changed the shape of tumor cells (Fig. 3).

Role of the Zeb1 gene in regulating the feedback of miR-23a. RT-qPCR was employed for real-time quantification detection of miR-23a in the OCM-1 cells in the Zeb1 overexpressed stably transfected group, the non-transfected control group, and blank transfected control group. The results showed that the average relative expression levels in the non-transfected control group, empty vector control group, and the Zeb1 overexpressed transfected group were 4.04, 4.13, and 1.34, respectively (Fig. 4A). The Zeb1 overexpressed transfection decreased the expression level of miR-23a in the OCM-1 cells by $\sim 67 \%$ relative to the control group, $p<0.05$. RT-qPCR was utilized to detect the relative expression levels of mRNA in the epithelium-derived marker E-cadherin, mesenchymal cell-labeled genes vimentin and $\mathrm{N}$-cadherin in the human choroidal melanoma OCM-1 cells of the Zeb1 gene overexpressed stable strains and the control groups (non-transfected control group and empty vector transfection control group). The average expression levels of E-cadherin in the non-transfected control group, empty vector transfection control group, and Zeb1 overexpressed transfected group were 1.09, 1, and 0.60 , respectively. The expression level of E-cadherin in the overexpressed transfection group decreased when compared with that in the control group, $\mathrm{p}<0.05$ (Fig. 4B). The average expression levels of vimentin in various groups were $1.18,1$, and 1.69 , respectively. The average expression levels of $\mathrm{N}$-cadherin were 1, 1.11, and 2.09. The average expression level in the Zeb1 overexpressed transfected group increased significantly when compared with that in the two control groups, $\mathrm{p}<0.05$. The differences between the blank control transfection group and the non-transfection group were not significant. Western blot analysis was used for quantitative analysis of the protein levels in E-cadherin, vimentin, and N-cadherin in the OCM-1 cells in the Zeb1 overexpression group and the two control groups. The result was basically consistent with the RT-qPCR result. The Zeb1 overexpression transfection decreased the expression level of the E-cadherin protein compared with that in 
A
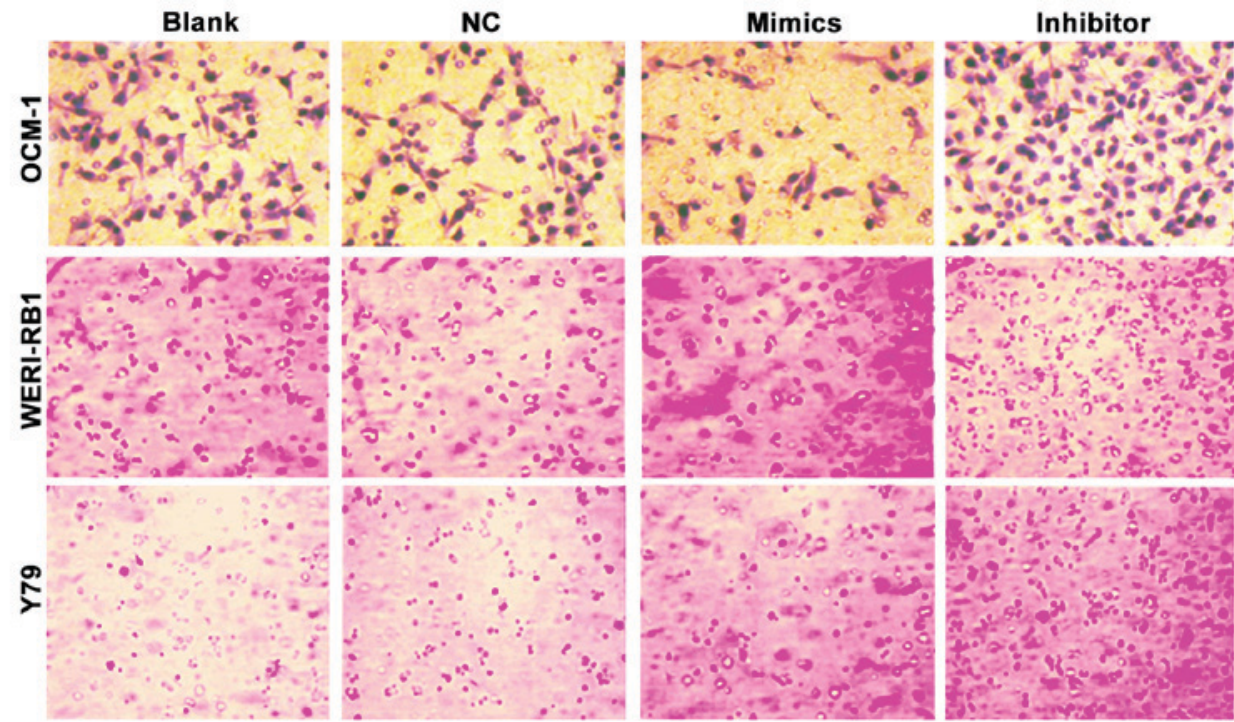

B

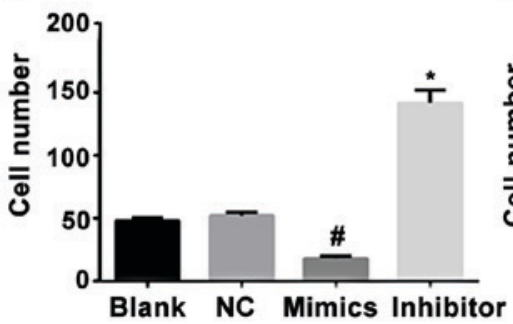

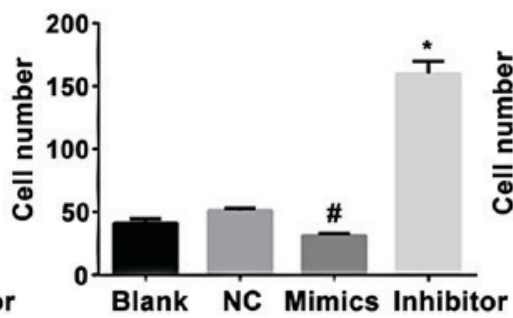

D

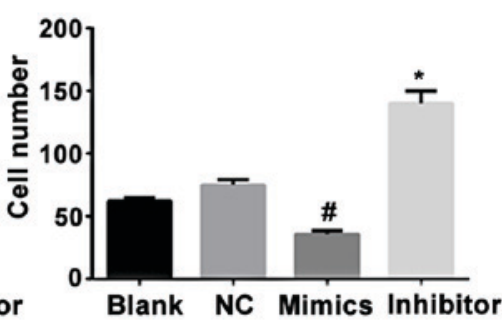

Figure 3. Effect of miR-23a transfection on the migration capacity of the intraocular tumor cells. (A) Optical microscope observation of the Transwell migrated cells of the choroidal melanoma cell strains OCM-1, retinoblastoma cell strains WERI-RB1 and Y79 transfected with miRNA-23a at $24 \mathrm{~h}$. Blank, non-transfection group; NC, negative control transfection group; Mimics, mimic transfection group; Inhibitor, inhibitor transfection group. (B-D) Statistics of cell migration of the cell strains (non-transfection group, negative control transfection group, mimic transfection group, inhibitor transfection group). The counts of the cells that permeated the membrane and entered the lower chamber in various groups transfected with miR-23a decreased significantly $(\# \mathrm{p}<0.05)$. The count of the cells that migrated to the lower chamber in the miR-23a inhibitor group increased significantly ( $\left.{ }^{*}<<0.05\right)$. ANOVA was used for comparison of multiple groups and the post hoc test was LSD.

the control group, whereas the expression levels of vimentin and $\mathrm{N}$-cadherin increased compared with that in the control group (Fig. 4C and D).

\section{Discussion}

Zeb1 is one of the important transcription factors in EMT. It is bound to E-box in the genetic transcription promoter sequence of the epithelial cell-labeled marker E-cadherin to inhibit transcription (20) and initiate EMT (21). Zeb1 is an essential factor for cells to maintain their mesenchyme characteristics. Normal epithelial tissue and low-malignant highly differentiated cancers tend to not or seldom express the factor. However, the Zebl gene is often highly expressed in poorly differentiated malignant tumors. In other solid tumor cell strains, miR-23a can directly act on and degrade Zeb1 thus eliminating its inhibition over E-cadherin, upregulating the expression of E-cadherin, reversing EMT, and producing mesenchymal epithelization (22). Furthermore, research also showed that the Zeb1 gene directly inhibits the E-cadherin gene via E-box. A study suggested that microRNA-23a suppressed CDH1 expression and promoted EMT (23). In this study, the results showed that the protein E-cadherin increased while the expression of the mesenchyme-labeled proteins of vimentin and $\mathrm{N}$-cadherin decreased in the mimics group. This may be caused by different cells used in different studies. Zeb1 has a negative feedback effect on miR-23a. They can form a negative feedback loop. The results showed that miR-23a and Zeb1 form a bidirectional inhibitory negative feedback loop, which plays an important role in regulating EMT. In addition, the compound formed by the Zeb1 protein and the histone deacetylase may participate in transcription and splicing of E-cadherin thus inhibiting its expression. miR-23a or b can inhibit the formation of such a compound thus promoting the expression of E-cadherin (24,25).

The expression of Zeb1 gene at the levels of mRNA and protein in the OCM-1, WERI-RB1, and Y79 cell strains transfected with miR-23a decreased significantly compared with that in the control group. Moreover, the expression of RNA and protein of the E-cadherin gene with epithelial cell specificity increased significantly. However, the expression of the mesenchyme-labeled proteins vimentin and $\mathrm{N}$-cadherin decreased due to inhibition. Mesenchymal-epithelial transition occurred in cells. On the contrary, among the three intraocular tumor cell strains in the miR-23a inhibitor transfection group, the expression level of Zeb1 increased significantly relative to the control group. The expression level of E-cadherin decreased. The expression levels of vimentin and $\mathrm{N}$-cadherin increased. EMT occurred in the cells. This conclusion is consistent with the result of multiple researches on tumor cells at other sites. 
A

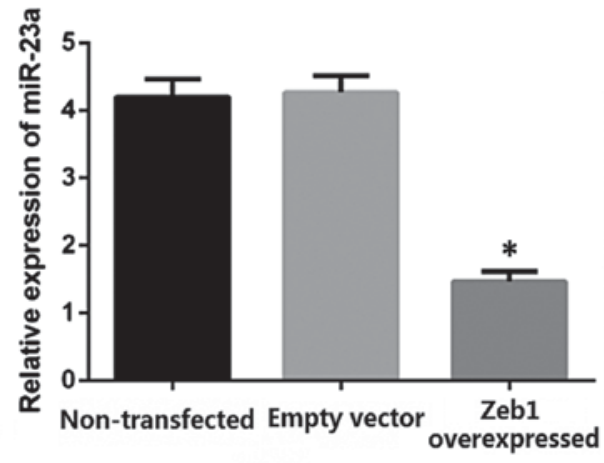

C

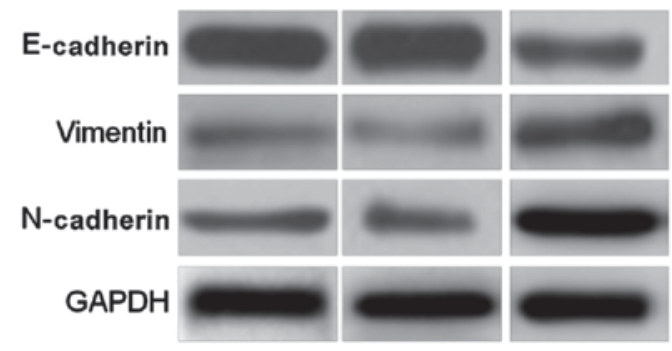

B

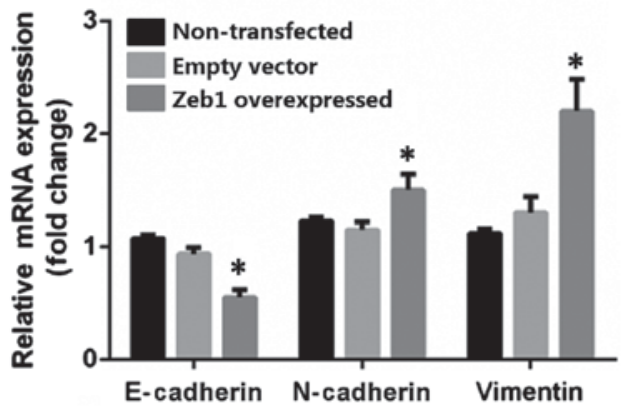

D

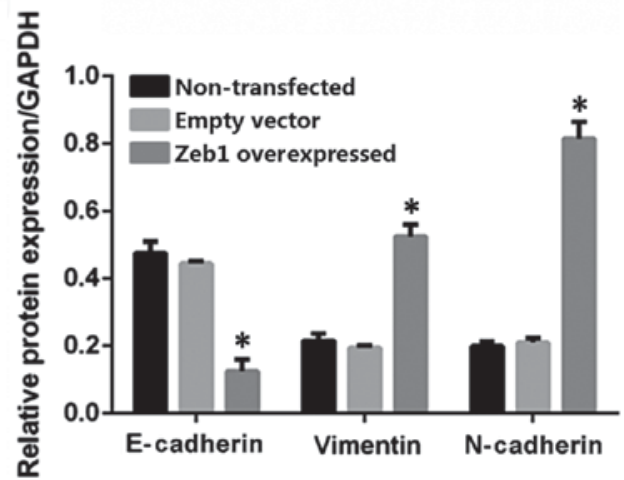

Figure 4. Role of the Zeb1 gene in regulating the feedback of miR-23a. (A) RT-qPCR detection of the effect of the Zeb1 overexpressed lentivirus stable transfection on the expression level of miR-23a in the OCM-1 cells. The Zeb1 overexpressed transfection enabled the relative expression level of miR-23a to decrease to $1 / 3$ of than before transfection. The differences between the Zeb1 overexpressed transfected group and the non-transfected control group were statistically significant ( $" \mathrm{p}<0.05)$. The expression level of miR-23a did not change in the empty vector control group. (B) RT-qPCR detection of the effect of the Zeb1 overexpressed lentivirus stable transfection on the genes related to the EMT in the human choroidal melanoma cell strains OCM-1. The stable transfection of the Zeb1 overexpressed lentivirus decreased the expression of mRNA of the epithelium-derived marker E-cadherin in the OCM-1 cells relative to the control group $\left({ }^{*} \mathrm{p}<0.05\right)$. The relative expression levels of the mesenchyme-deprived markers vimentin and N-cadherin increased significantly ( $\left.\mathrm{k} p<0.05\right)$. (C and D) Western blot analysis of the role of the Zeb1 overexpressed lentivirus stable transfection in regulating the epithelium and mesenchyme-labeled proteins in the OCM-1 cells. The Zeb1 overexpressed stable transfection decreased the expression level of the epithelial cell marked protein E-cadherin in the OCM-1 cells relative to the two control groups. It increased the expression level of the mesenchyme-labeled proteins vimentin and N-cadherin ("p<0.05). ANOVA was used for comparison of multiple groups and the post hoc test was LSD. EMT, epithelial-mesenchymal transition.

We also detected evidence for epithelium-mesenchyme mutual transition in the intraocular tumor cell strains.

We analyzed the expression level of miR-23a, the epithelial marker E-cadherin and mesenchyme marker vimentin in nine groups of cells (groups of non-transfected and transfected intraocular tumor cell strains) and their ratio. It indicated that miR-23a and the ratio of E-cadherin RNA and protein expression, E-cadherin, and vimentin protein are positively correlated. However, miR-23a and the expression level of the vimentin protein are negatively correlated. It further demonstrated the roles of miR-23a in promoting mesenchyme epithelization and inhibiting EMT. However, the sample size in this study is excessively small and deviation is also introduced to the effect of different transfection intervention on EMT. Therefore, the relevant significance obtained in this chapter can only be considered as a prediction of the trend of the function of EMT in the three intraocular tumors based on miR-23a.

The Transwell experiment was used to detect the effect of the changes in the expression level of miR-23a on the migration capacity of the three strains of tumor cells. It was found that miR-23a can significantly inhibit the migration capacity of these tumor cells. Inhibition of miR-23a changed the shape of tumor cells. The capacity of the cells to permeate the semipermeable membrane in the mimic transfection group decreased significantly. The cells in the inhibitor transfection group exhibited stronger capacity of motion and migration. In combination with the detection results of epithelial and mesenchymal markers of the cells in various groups, the changes in motion and migration capacity of the tumor cells can be associated with EMT. EMT can significantly strengthen the migration capacity of the cells while mesenchymal-epithelial transition significantly decreases the cellular migration capacity. The mesenchyme transition process enables the epithelium-derived tumor cells to lose the original closely-linked proteins and produce cytoskeletal proteins of mesenchymal cells. They are more likely to change their morphology and have stronger motion and transmembrane migration capacity. Therefore, miR-23a probably changes the motion capacity of the cells regulating the EMT of the tumor cells.

The human choroidal melanoma cell strains OCM-1 were subjected to puromycin resistance screening at $72 \mathrm{~h}$ after transfected with the Zeb1 overexpressed lentivirus particles to obtain the stable transfected OCM-1 cell strains. RT-qPCR detection has found that the expression of miR-23a in the tumor cells is significantly inhibited, indicating that Zeb1 plays a role in inhibiting the feedback of miR-23a. Both RT-qPCR and western blot analysis showed that the expression of the epithelial cell gene E-cadherin in the OCM-1 cells following Zeb1 overexpressed stable transfection is downregulated. The expression of the genes of vimentin and $\mathrm{N}$-cadherin related to 
mesenchymal cells is upregulated. It demonstrates the role of Zeb1 in promoting EMT. It suggests that a negative feedback regulation loop with miR-23a/Zeb1 mutual inhibition occurs in the intraocular malignant tumor cell strains, which coincides with the results of the experiments on other human tumors. It demonstrates that mutual transition between epithelial and mesenchymal cells can occur in the intraocular malignant tumor cells. miR-23a can induce mesenchymal-epithelial transition in the tumor cells whereas Zeb1 can promote EMT. The tumorigenic ability of these tumor cells may increase with the increase of EMT.

The mechanism of miR-23a regulating Zeb1 is still unknown. It may be related to binding to 3'UTR. However, the Zeb1 3'UTR reporter gene was not done because only locus analysis was used and the results of interfering miR-23a and overexpressing Zeb1 should be supported by interfering Zeb1 and overexpressing miR-23a. Such tests will be carried out in the future. The expression of miR-23a and Zeb1 should be recorded at different time-points to test the 'feedback'. Due to the cost of the experiment, this experiment does not take into account the process of multiple time-points repeated verification, so this project only discusses the problem of specific time.

In conclusion, EMT is detected in the human intraocular malignant tumor cells (choroidal melanoma cell strains OCM-1, retinoblastoma cell strains WERI-RB1 and Y79). The report verifies the presence of the bidirectional negative feedback regulation loop of miR-23a/Zebl and the role of the pathway in regulating EMT in tumor cells and regulating the migration capacity of tumor cells in vitro in the intraocular tumor system.

\section{Acknowledgements}

Not applicable.

\section{Funding}

No funding was received.

\section{Availability of data and materials}

The datasets used and/or analyzed during the present study are available from the corresponding author on reasonable request.

\section{Authors' contributions}

YW and HZ conceived and designed the study, and drafted this paper. YW, YL and WG collected, analyzed and interpreted the experiment data, and revised the manuscript critically for important intellectual content. All authors have read and approved the final manuscript.

\section{Ethics approval and consent to participate}

The study was approved by the Ethics Committee of the Affiliated Hospital of Inner Mongolia Medical University (Hohhot, Inner Mongolia, China).

\section{Patient consent for publication}

Not applicable.

\section{Competing interests}

The authors declare that they have no competing interests.

\section{References}

1. Cho MH, Park JH, Choi HJ, Park MK, Won HY, Park YJ, Lee CH, Oh SH, Song YS, Kim HS, et al: DOT1L cooperates with the c-Myc-p300 complex to epigenetically derepress CDH1 transcription factors in breast cancer progression. Nat Commun 6: $7821,2015$.

2. Ichikawa K, Kubota Y, Nakamura T, Weng JS, Tomida T, Saito $\mathrm{H}$ and Takekawa M: MCRIP1, an ERK substrate, mediates ERK-induced gene silencing during epithelial-mesenchymal transition by regulating the co-repressor CtBP. Mol Cell 58: 35-46, 2015.

3. Wang L, Yang H, Abel EV, Ney GM, Palmbos PL, Bednar F, Zhang Y, Leflein J, Waghray M, Owens S, et al: ATDC induces an invasive switch in KRAS-induced pancreatic tumorigenesis. Genes Dev 29: 171-183, 2015.

4. Chen L, Gibbons DL, Goswami S, Cortez MA, Ahn YH, Byers LA, Zhang X, Yi X, Dwyer D, Lin W, et al: Metastasis is regulated via microRNA-200/ZEB1 axis control of tumour cell PD-L1 expression and intratumoral immunosuppression. Nat Commun 5: 5241, 2014.

5. Wang Y, Bu F, Royer C, Serres S, Larkin JR, Soto MS, Sibson NR, Salter V, Fritzsche F, Turnquist C, et al: ASPP2 controls epithelial plasticity and inhibits metastasis through $\beta$-catenin-dependent regulation of ZEB1. Nat Cell Biol 16: 1092-1104, 2014.

6. Zhang P, Wei Y, Wang L, Debeb BG, Yuan Y, Zhang J, Yuan J, Wang M, Chen D, Sun Y, et al: ATM-mediated stabilization of ZEB1 promotes DNA damage response and radioresistance through CHK1. Nat Cell Biol 16: 864-875, 2014.

7. Chao $\mathrm{CH}$, Chang $\mathrm{CC}$, Wu MJ, Ko HW, Wang D, Hung MC, Yang JY and Chang CJ: MicroRNA-205 signaling regulates mammary stem cell fate and tumorigenesis. J Clin Invest 124: 3093-3106, 2014.

8. Park KS, Raffeld M, Moon YW, Xi L, Bianco C, Pham T, Lee LC, Mitsudomi T, Yatabe Y, Okamoto I, et al: CRIPTO1 expression in EGFR-mutant NSCLC elicits intrinsic EGFR-inhibitor resistance. J Clin Invest 124: 3003-3015, 2014.

9. Yuan JH, Yang F, Wang F, Ma JZ, Guo YJ, Tao QF, Liu F, Pan W, Wang TT, Zhou CC, et al: A long noncoding RNA activated by TGF- $\beta$ promotes the invasion-metastasis cascade in hepatocellular carcinoma. Cancer Cell 25: 666-681, 2014.

10. Lehmann W, Mossmann D, Kleemann J, Mock K, Meisinger C, Brummer T, Herr R, Brabletz S, Stemmler MP and Brabletz T: ZEB1 turns into a transcriptional activator by interacting with YAP1 in aggressive cancer types. Nat Commun 7: 10498, 2016.

11. Zheng X, Carstens JL, Kim J, Scheible M, Kaye J, Sugimoto H, Wu CC, LeBleu VS and Kalluri R: Epithelial-to-mesenchymal transition is dispensable for metastasis but induces chemoresistance in pancreatic cancer. Nature 527: 525-530, 2015.

12. Yang Y, Ahn YH, Chen Y, Tan X, Guo L, Gibbons DL, Ungewiss C, Peng DH, Liu X, Lin SH, et al: ZEB1 sensitizes lung adenocarcinoma to metastasis suppression by PI3K antagonism. J Clin Invest 124: 2696-2708, 2014.

13. Tian K, Di R and Wang L: MicroRNA-23a enhances migration and invasion through PTEN in osteosarcoma. Cancer Gene Ther 22: 351-359, 2015.

14. Wen YC, Lee WJ, Tan P, Yang SF, Hsiao M, Lee LM and Chien MH: By inhibiting snail signaling and miR-23a-3p, osthole suppresses the EMT-mediated metastatic ability in prostate cancer. Oncotarget 6: 21120-21136, 2015.

15. Tang J, Zhao H, Cai $\mathrm{H}$ and $\mathrm{Wu} \mathrm{H}$ : Diagnostic and prognostic potentials of microRNA-27a in osteosarcoma. Biomed Pharmacother 71: 222-226, 2015.

16. Zhang XW, Liu N, Chen S, Wang Y, Zhang ZX, Sun YY, Qiu GB and $\mathrm{Fu}$ WN: High microRNA-23a expression in laryngeal squamous cell carcinoma is associated with poor patient prognosis. Diagn Pathol 10: 22, 2015.

17. Ma G, Dai W, Sang A, Yang X and Gao C: Upregulation of microRNA-23a/b promotes tumor progression and confers poor prognosis in patients with gastric cancer. Int J Clin Exp Pathol 7: 8833-8840, 2014.

18. He Y, Meng C, Shao Z, Wang H and Yang S: MiR-23a functions as a tumor suppressor in osteosarcoma. Cell Physiol Biochem 34: 1485-1496, 2014. 
19. Yang L, Yang X, Ji W, Deng J, Qiu F, Yang R, Fang W, Zhang L, Huang D, Xie C, et al: Effects of a functional variant c.353T $>C$ in snail on risk of two contextual diseases. Chronic obstructive pulmonary disease and lung cancer. Am J Respir Crit Care Med 189: 139-148, 2014.

20. Li C, Ma H, Wang Y, Cao Z, Graves-Deal R, Powell AE, Starchenko A, Ayers GD, Washington MK, Kamath V, et al: Excess PLAC8 promotes an unconventional ERK2-dependent EMT in colon cancer. J Clin Invest 124: 2172-2187, 2014.

21. Caramel J, Papadogeorgakis E, Hill L, Browne GJ, Richard G, Wierinckx A, Saldanha G, Osborne J, Hutchinson P, Tse G, et al: A switch in the expression of embryonic EMT-inducers drives the development of malignant melanoma. Cancer Cell 24 466-480, 2013.

22. Hur K, Toiyama Y, Takahashi M, Balaguer F, Nagasaka T, Koike J, Hemmi H, Koi M, Boland CR and Goel A: MicroRNA-200c modulates epithelial-to-mesenchymal transition (EMT) in human colorectal cancer metastasis. Gut 62: 1315-1326, 2013.
23. Cheng L, Yang T, Kuang Y, Kong B, Yu S, Shu H, Zhou H and $\mathrm{Gu}$ J: MicroRNA-23a promotes neuroblastoma cell metastasis by targeting CDH1. Oncol Lett 7: 839-845, 2014.

24. Celià-Terrassa T, Meca-Cortés $\mathrm{O}$, Mateo F, Martínez de Paz A, Rubio N, Arnal-Estapé A, Ell BJ, Bermudo R, Díaz A, Guerra-Rebollo M, et al: Epithelial-mesenchymal transition can suppress major attributes of human epithelial tumor-initiating cells. J Clin Invest 122: 1849-1868, 2012.

25. Mizuguchi Y, Isse K, Specht S, Lunz JG III, Corbitt N, Takizawa T and Demetris AJ: Small proline rich protein $2 \mathrm{a}$ in benign and malignant liver disease. Hepatology 59: 1130-1143, 2014.

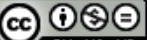

This work is licensed under a Creative Commons Attribution-NonCommercial-NoDerivatives 4.0 International (CC BY-NC-ND 4.0) License. 